\title{
A Strategy for the Generation of an Infectious Transmissible Gastroenteritis Coronavirus from Cloned cDNA
}

\author{
FERNANDO ALMAZAN, JOSE M. GONZALEZ, ZOLTAN PENZES, \\ ANDER IZETA, ENRIQUE CALVO, AND LUIS ENJUANES \\ Centro Nacional de Biotecnología, CSIC, Department of Molecular and Cell Biology, Campus \\ Universidad Autónoma, Cantoblanco, 28049 Madrid, Spain
}

\section{INTRODUCTION}

To date reverse genetics of coronavirus has been possible by targeted recombination following the procedure initially developed by Masters' group (Koetzner et al., 1992). However, the construction of a full-length cDNA clone, from which infectious RNA may be transcribed, will considerably improve the genetic manipulation of coronaviruses. Unfortunately, the size of the coronavirus genome and the instability in bacteria of plasmids carrying coronavirus replicase sequences have hampered the construction of a full-length cDNA clone (Masters, 1999). To overcome these problems we have combined three strategies: (i) a two-step amplification system that couples transcription in the nucleus from the cytomegalovirus (CMV) promoter with a second amplification step in the cytoplasm by the viral polymerase; (ii) the construction of the full-length cDNA from a defective minigenome (DI) that was stably and efficiently replicated by the helper virus (Izeta et al., 1999); and (iii) the full-length cDNA was cloned as a bacterial artificial chromosome (BAC), a low-copy number plasmid, which is present in one or two copies per cell.

In the present study, we report the recovery of infectious transmissible gastroenteritis virus (TGEV) from cloned cDNA and show that this procedure can be used to generate a genetically modified TGEV.

The Nidoviruses (Coronaviruses and Arteriviruses).

Edited by Ehud Lavi et al., Kluwer Academic/Plenum Publishers, 2001. 


\section{MATERIALS AND METHODS}

\subsection{Cells, Viruses, Plasmid and Bacteria Strains}

Epithelial swine testis (ST) cells were kindly provided by L. Saif (Ohio State University, OH). The TGEV strain PUR46-MAD (PUR-MAD) and PUR46-C11 (PUR-C11) (Sánchez et al., 1999) were grown and titered as described (Sánchez et al., 1990).

Plasmid pBeloBAC11 (Wang et al., 1997) was kindly provided by $\mathrm{H}$. Shizuya and M. Simon (California Institute of Technology, Pasadena, CA). E. coli $\mathrm{DH} 10 \mathrm{~B}$ strain was obtained from GIBCO/BRL.

\subsection{Construction of the TGEV Full-Length cDNA}

As a backbone for the construction of a full-length cDNA clone of the TGEV, the minigenome DI-C, derived of the PUR-MAD strain, was used (Izeta et al., 1999). DI-C RNA has three deletions ( $\Delta 1, \Delta 2$, and $\Delta 3$ ) within ORFs $1 \mathrm{a}, 1 \mathrm{~b}$, and between genes $S$ and 7 , respectively. These deletions were restored by cloning a set of cDNA fragments generated by standard RT-PCR techniques, using the medium copy number plasmid pACNR1180. Deletions $\Delta 2$ and $\Delta 3$ were restored generating a stable plasmid. However, when the deletion $\Delta 1$ was completed, the resultant plasmid was unstable within the bacteria, and only mutated forms were recovered. To overcome this toxicity problem, TGEV cDNAs were cloned in pBeloBAC 11 leading to plasmids pBAC-TGEV ${ }^{\text {Clal }}$, containing a $5.2 \mathrm{~kb}$ ClaI-Clal fragment from nucleotides 4,417 to 9,615 , and pBAC$\mathrm{TGEV}^{\Delta C l a \mathrm{l}}$, encoding the rest of the TGEV genome (Almazan et al., 2000). The last step for the generation of the full-length cDNA (pBAC$\mathrm{TGEV}^{\mathrm{FL}}$ ) consisted of the insertion of de ClaI-ClaI fragment into ClaIlinearized pBAC-TGEV ${ }^{\Delta C l a l}$ (Fig. 1).

The full-length cDNA was under the control of the CMV promoter and flanked at its 3' end by a 24 bp poly(A) tail followed by the hepatitis delta virus ribozyme and the bovine growth hormone termination and polyadenylation sequences (Izeta et al., 1999). Details of this construction are presented in the report entitled "Cloning of a Transmissible Gastroenteritis Coronavirus Full-Length cDNA“, also published in this book.

\subsection{Recovery of Infectious TGEV from the cDNA}

ST cells $\left(10^{6}\right)$ were grown to $60 \%$ confluence and were transfected with $10 \mu \mathrm{g}$ of either pBAC-TGEV ${ }^{\mathrm{FL}}$ or pBAC-TGEV ${ }^{\mathrm{FL}-(\text { Clal)RS }}$ (pBAC- 
$\mathrm{TGEV}^{\mathrm{FL}}$ plasmid carrying the ClaI-ClaI fragment in the reverse-sense) using $15 \mu \mathrm{g}$ of Lipofectine (Life Technologies, GIBCO) according to the manufacture's specifications. After an incubation period of 2 days, the cell supernatant (referred to as passage 0 ) was passaged six times on fresh ST cells and the presence of virus was analyzed by plaque titration. After six passages the virus was cloned by three plaque purification steps.

\section{$2.4 \quad$ Virulence Assay}

The in vivo growth and virulence of TGEV isolates and the recovered virus were determined as described (Sánchez et al., 1999). The virus titers in lung, jejunum, and ileum were determined 2 days after infection.

\section{RESULTS AND DISCUSSION}

\subsection{Rescue of an Infectious TGEV from a cDNA Clone}

To obtain a cDNA encoding a full-length TGEV RNA, a cDNA encoding the TGEV derived DI-C was used as the starting point. The three deletions that DI-C has in relationship to the parental virus PURMAD were restored (see Materials and Methods) and the full-length

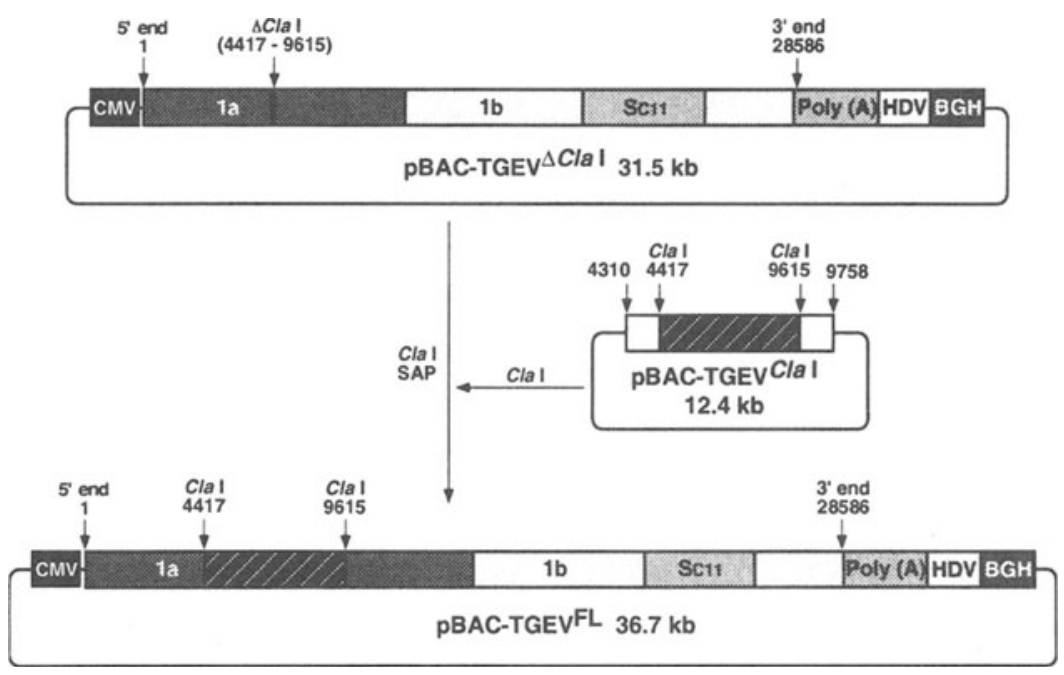

Figure 1. Cloning of the TGEV cDNA in pBeloBAC11. Plasmid pBAC-TGEV ${ }^{\mathrm{FL}}$ were generated as described in Materials and Methods. CMV, cytomegalovirus immediateearly promoter; Poly(A), tail of 24 A residues; HDV, hepatitis delta virus ribozyme; BGH, bovine growth hormone termination and polyadenylation sequences; SC11, S gene of PUR-C11 strain; SAP, shrimp alkaline phosphatase. 
cDNA was cloned as a BAC (Fig. 1) (Almazán et al., 2000). The resulting plasmid, pBAC-TGEV ${ }^{\mathrm{FL}}$, was stable for at least 80 generations in $\mathrm{DH} 10 \mathrm{~B}$ cells. In addition, to generate a cDNA encoding a fully active TGEV that would replicate both within the enteric and the respiratory tracts and preserve the virulence of the original in vivo isolates, the spike (S) gene of the PUR-MAD strain, which replicates abundantly within the respiratory tract and scarcely in the enteric tract of swine, was replaced by the S gene of PUR-C11 strain, which replicates with high titers within both the respiratory and the enteric tracts (Sánchez et al., 1999).

To recover an infectious TGEV from the cDNA clone, ST cells were transfected with plasmid $\mathrm{pBAC}-\mathrm{TGEV}^{\mathrm{FL}}$, and the cell supernatant was passaged six additional times. Virus titers quickly increased with passage and were around $10^{8} \mathrm{pfu} / \mathrm{ml}$ by passage 4 . However, in the mocktransfected cultures or in cells transfected with the same plasmid but carrying the ClaI fragment in the reverse-sense (pBAC-TGEV ${ }^{\mathrm{FL}-(\mathrm{ClaI}) \mathrm{RS}}$ ) no virus was recovered (Fig. 2). After six passages, the virus was cloned, and the selected virus was named rPUR-MAD-SC11.

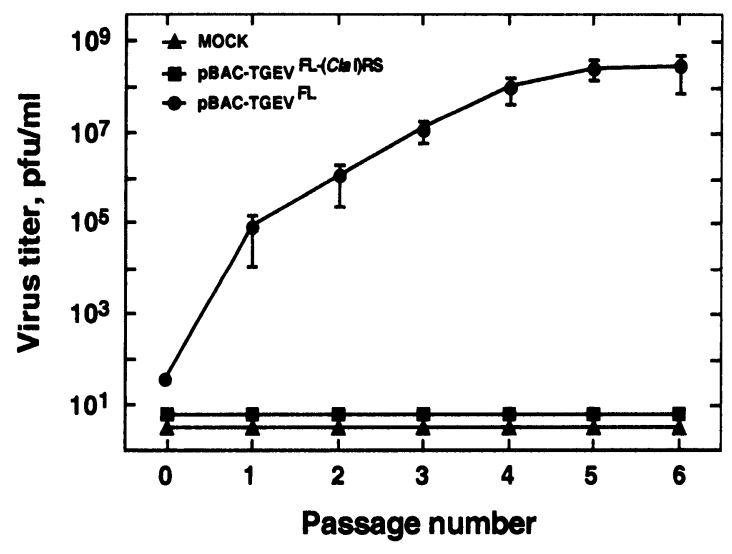

Figure 2. Infectious TGEV recovered from cDNA. After transfection, the recovered virus was passaged, and the culture supernatant were titrated on ST cells. Error bars represent standard deviations of the mean from six experiments.

The rescued virus conserved all the genetics markers introduced throughout the sequence and showed the antigenic characteristics expected for the synthetic virus. The cytopathic effects produced by the rescued virus included induction of cell fusion and formation of large size plaques. These characteristics are identical to those of the parental virus, which provided the $\mathrm{S}$ gene, and not to the PUR-MAD strain, which provided the rest of the genome, suggesting that the $\mathrm{S}$ gene is a determinant of cell fusion and plaque morphology. 
Since the cDNA was transcribed within the nucleus, we investigated if there was splicing of the genomic RNA during its translocation from the nucleus to the cytoplasm. For this purpose, the sequences with the highest splicing potential along the TGEV sequence were determined, and the RNA fragments with the potential splice sites were amplified by RTPCR by using as template the cytoplasmic RNA at passage 0 and 1. Splicing was observed in only one amplified fragment, and only $20 \%$ of the molecules were spliced. Interestingly, the genome with no splicing was favored by selection after one passage. To asses definitively whether splicing had taken place in the viral RNA selected during virus replication, we determined the full-length sequence of the cloned virus. Splicing was not detected and only five nucleotide differences were observed between the sequence of the rescued RNA genome and that of the cDNA clone.

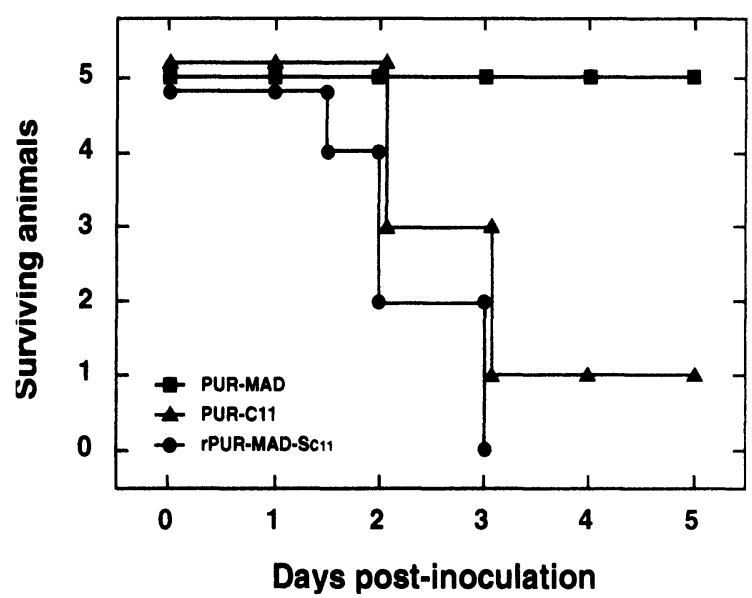

Figure 3. Surviving newborn minipigs infected with rPUR-MAD-SCI1, PUR-MAD, or PUR-C11 at $48 \mathrm{~h}$ after birth with $2 \times 10^{8}$ pfu per animal.

\subsection{Tropism and Virulence of the Infectious cDNA}

The virulence and tropism of clone rPUR-MAD-SC11 have been analyzed during infection of breast-fed newborn animals. rPUR-MADSC11 showed a mortality of $100 \%$, similar to that of the parental virus that provided the $\mathrm{S}$ gene (PUR-C11). In contrast, the parental virus providing all of the genes except the $S$ gene (PUR-MAD) produced no clinical signs, as expected (Fig. 3). On the other hand, the rPUR-MAD$\mathrm{SCl} 1$ virus grew in the jejunum and ileum of infected animals to titers as high as those of the parental enteric virus (PUR-C11), whereas the parental virus PUR-MAD produced low titers. Both parental viruses and 
the rescued one grew very well in the lungs. These data indicated that the $\mathrm{S}$ gene is a determinant of TGEV tropism and virulence.

\section{CONCLUSIONS}

A fully functional infectious cDNA clone, leading to a virulent TGEV that replicates both in the enteric and the respiratory tracts, has been engineered. This cDNA clone will have an important impact on the study of mechanisms of coronavirus replication and transcription and provides an invaluable tool for the experimental investigation of virus-host interactions.

\section{ACKNOWLEDGMENTS}

This research was supported by grants from the Comisión Interministerial de Ciencia y Tecnología (Spain), the Community of Madrid, the European Community (Biotechnology, FAIR and Control of Infectious Diseases Programs), and Fort Dodge Veterinaria (Spain).

\section{REFERENCES}

Almazán, F., González, J. M., Pénzes, Z., Izeta, A., Calvo, E., Plana-Durán, J. and Enjuanes, L. 2000. Engineering the largest RNA virus genome as an infectious bacterial artificial chromosome. Proc. Natl. Acad. Sci. U. S. A. 97:5516-5521.

Izeta, A., Smerdou, C., Alonso, S., Penzes, Z., Méndez, A., Plana-Durán, J. and Enjuanes, L. 1996. Replication and packaging of transmissible gastroenteritis coronavirusderived synthetic minigenomes. J. Virol 73: 1535-1545.

Koetzner, C. A., Parker, M. M., Ricard, C. S., Sturman, L. S. and Masters, P. S. 1992. Repair and mutagenesis of the genome of a deletion mutant of the coronavirus mouse hepatitis virus by targeted RNA recombination. J. Virol. 66: 1841-1848.

Masters, P. S. 1999. Reverse genetics of the largest RNA viruses. Adv. Virus Res. 53: 245 264.

Sánchez, C. M., Jiménez, G., Laviada, M. D., Correa, I., Suñé, C., Bullido, M. J., Gebaguer, F., Smerdou, C., Callebaut, P. et al. 1990. Antigenic homology among coronaviruses related to transmissible gastroenteritis virus. Virology 174: 410-417.

Sánchez, C. M., Izeta, A., Sánchez-Morgado, J. M., Alonso, S., Sola, I., Balasch, M., PlanaDurán, J. and Enjuanes, L. 1999. Targeted recombination demonstrates that the spike gene of transmissible gastroenteritis coronavirus is a determinant of its enteric tropism and virulence. J. Virol. 73: 7607-7618.

Wang, K., Boysen, C., Shizuya, H., Simon, M. I. and Hood, L. 1997. Complete nucleotide sequence of two generations of a bacterial artificial chromosome cloning vector. Biotechniques 23: 992-994. 